\title{
WEIERSTRASS POINTS AND ANALYTIC SUBMANIFOLDS OF TEICHMUELLER SPACE ${ }^{1}$
}

\author{
H. M. FARKAS
}

I. A few years ago, H. E. Rauch [2], and more recently the present author [1] proved the following

TheOREM. The locus of $T^{o}(S)$ possessing a Weierstrass point whose Weierstrass sequence begins with a fixed $r<g$ is a complex-analytic submanifold of $T^{\circ}(S)$ of complex dimension $r+2 g-3$ when $r+1$ is a gap, and is a subvariety of complex dimension $r+2 g-4$ when $r+1$ is not a gap.

When Rauch originally proved this theorem, he mentioned that it was not altogether clear that the singularity in the exceptional cases of the theorem is genuine and not a product of the method of proof. The present author's proof seemed to lend supporting evidence to the fact that the singularity is indeed genuine.

The purpose of this paper is to point out that for $g \geqq 4$ and $r=3$ the exceptional case does not occur. We begin with a brief summary of the pertinent results from the theory of compact Riemann surfaces. In what follows, $S$ will always denote a compact Riemann surface of genus $g$.

There are exactly $g$ orders $n_{i}, 0 \leqq n_{1}<\cdots<n_{g} \leqq 2 g$, that can be specified at each point $p \in S$ such that no nonconstant meromorphic function exists on $S$ whose only singularity is a pole of order $n_{i}$ at $p$. Given a general point $p \in S$ its gap sequence is $(1,2, \cdots, g)$; however, there do exist points on $S$ whose gap sequence omits some of these numbers. These points are called Weierstrass points. In other words, the gap sequence for a Weierstrass point omits an integer $n, 2 \leqq n \leqq g$. When we have a surface with a Weierstrass point with $n=2$, the surface is said to be hyperelliptic. It is well known that a hyperelliptic surface has $2 g+2$ Weierstrass points and for each one of them the gap sequence is $(1,3, \cdots, 2 g-1)$. The complement of the gap sequence in the sequence of integers $(0,1,2, \cdots, 2 g)$ is called the Weierstrass sequence. In this paper we are concerned with those compact Riemann surfaces of genus $g, g \geqq 4$, possessing a Weierstrass point whose Weierstrass sequence begins with $n=3$.

\footnotetext{
Presented to the Society, January 24, 1968; received by the editors September 13, 1967.
}

${ }^{1}$ Research partially supported by NSF GP6658. 
An integral divisor on $S$ is a set of points and associated nonnegative integers $p_{1}^{\alpha} \cdots p_{n}^{\alpha} \cdot \sum_{k=1}^{n} \alpha_{k}=d$ is called the degree of the divisor. If $f$ is a meromorphic function on $S$, denote by $p_{1}^{\alpha} \cdots p_{r}^{\alpha}$ r and $Q_{1}^{\beta_{1}} \cdots Q_{k^{k}}^{\beta_{k}}$ the respective zeros and poles of $f$, the superscripts denoting multiplicities. The divisor of $f$ is defined to be $p_{1}^{\alpha_{1}} \ldots$ $p_{r}^{\alpha} Q_{1}^{-\beta_{1}} \cdots Q_{\mathbf{k}}^{-\beta_{k}}$. Since a meromorphic function $f$ on $S$ has the same number of zeros as poles, the degree of the divisor of $f$ is zero. The divisor of an Abelian differential of first kind is defined in an analogous manner.

The Riemann-Roch theorem [3] states that if $\zeta$ is an integral divisor of degree $r, l(1 / \zeta)=r-g+1+i(\zeta)$ where $l(1 / \zeta)$ is defined to be the dimension of the vector space of meromorphic functions whose divisors are multiples of $1 / \zeta$ and $i(\zeta)$ is defined to be the dimension of the space of Abelian differentials of first kind whose divisors are multiples of $\zeta$.

Suppose $\zeta$ is an integral divisor, degree $\zeta<g$, and $l(1 / \zeta)>1$. Then $\zeta$ is said to be a special divisor. For special divisors, Clifford's theorem [4] states that $i(\zeta) \leqq g-1 / 2$ degree $\zeta$. Furthermore, in the case of equality, the surface is hyperelliptic. Actually, Clifford's theorem is generally stated as a theorem on special linear series of degree $n$ and dimension $r\left(g_{n}^{r}\right)$; however, a simple application of the RiemannRoch theorem gives the form here stated.

The theorem we wish to prove will follow from the general facts stated above.

II. Lemma 1. Let $p$ be a Weierstrass point on $S$ whose Weierstrass sequence begins with 3 . Then $l\left(1 / p^{3}\right)=2$.

Proof. By hypothesis, there exists a meromorphic function $f$ on $S$ whose only singularity is a pole of order 3 at $p$. Hence $\{1, f\} \in L\left(1 / p^{3}\right)$, where $L\left(1 / p^{3}\right)$ is the vector space of meromorphic functions whose divisors are multiples of $1 / p^{3}$. If $l\left(1 / p^{3}\right)=3$, by the Riemann-Roch theorem, $3=3-g+1+i\left(p^{3}\right)$ or $i\left(p^{3}\right)=g-1$. This of course implies that $i\left(p^{2}\right)=g-1$ which, by another application of Riemann-Roch, implies the existence of a meromorphic function with a second order pole at $p$. This would contradict the hypothesis that the Weierstrass sequence begins with 3 .

LеммA 2. If the Weierstrass sequence at $p$ begins with 3 and, if 4 is not a gap, then $l\left(1 / p^{4}\right)=3$.

Proof. $L\left(1 / p^{4}\right) \supset\{1, f, g\}$ where $g$ is a function whose only singularity is a pole of order 4 at $p$. The fact that $l\left(1 / p^{4}\right)<4$ follows in the same way that we've shown in Lemma 1 that $l\left(1 / p^{3}\right)<3$. The 
proof is complete providing we make the trivial observation that $\{1, f, g\}$ are linearly independent.

LEMмA 3. If the Weierstrass sequence at p begins with 3, and if 4 is not a gap, then $S$ is hyperelliptic.

Proof. By Lemma $2 l\left(1 / p^{4}\right)=3$. By the Riemann-Roch theorem, $l\left(1 / p^{4}\right)=4-g+1+i\left(p^{4}\right)=3$ which implies that $i\left(p^{4}\right)=g-2$. Hence, if $g \geqq 5$ we have produced a special divisor $p^{4}$ of degree 4 with $i\left(p^{4}\right)$ $=g-1 / 2 \operatorname{deg}\left(p^{4}\right)=g-2$. By Clifford's theorem $S$ is hyperelliptic. If $g=4$, we have a divisor of degree 4 and index 2 . Therefore, there are two linearly independent Abelian differentials of first kind whose divisors are multiples of $p^{4}$. Denote these differentials by $w_{1}$ and $w_{2}$. $w_{1} / w_{2}$ is then a meromorphic function on $S$ with precisely two poles which implies hyperellipticity.

Theorem 1. Let $S$ be a compact Riemann surface of genus $g \geqq 4$. Let $p$ be a Weierstrass point on $S$ whose Weierstrass sequence begins with 3. Then 4 is a gap.

Proof. By Lemma 3, if 4 were not a gap, then $S$ would be hyperelliptic. Therefore $S$ would have $2 g+2$ Weierstrass points whose sequences all are $(2,4, \cdots, 2 g)$ contradicting the fact that there is a Weierstrass point whose Weierstrass sequence begins with 3 .

TheOREM 2. The locus of $T^{\circ}(S), g \geqq 4$, possessing a Weierstrass point whose Weierstrass sequence begins with 3 is $2 g$ complex dimensional complex analytic submanifold of $T^{\circ}(S)$.

Proof. The exceptional case having been ruled out, the proof is the same as in [1] or [2].

We end with the following remark: In [1], [2] it was shown that the exceptional case does not occur when $r=2$, confirming the wellknown fact that the hyperelliptic surfaces are a $2 g-1$ complex dimensional complex analytic submanifold of $T^{o}(S)$. Here we have shown that the exceptional case does not occur when $r=3$ and $g \geqq 4$. It seems quite likely though that for $r>3$ the singularity is indeed quite genuine.

ADDED IN PROOF. Theorem 1 can of course be proved without resorting to Clifford's theorem. It is an almost immediate consequence of the Weierstrass gap theorem. We have chosen the proof given above to show the more general fact that given a special divisor of degree 3 , say $\zeta$, such that $i(\zeta)=g-2$, then for any point $p \in S i(p \zeta)$ $=g-3$ unless $S$ is hyperelliptic. 


\section{REFERENCES}

1. H. M. Farkas, Special divisors and analytic subloci of Teichmueller space, Amer. J. Math. 88 (1966), 881-901.

2. H. E. Rauch, Weierstrass points, branch points, and moduli of Riemann surfaces, Comm. Pure Appl. Math. 12 (1959), 543-560.

3. G. Springer, Introduction to Riemann surfaces, Addison-Wesley, Reading, Mass., 1957.

4. J. G. Semple and L. Roth, Introduction to algebraic geometry, Clarendon, Oxford, 1949.

JOHNS HOPKINS UNIVERSITY 